Median NIHSS score at 24 hours postprocedure was 3 (IQR 1-12). Thirty-eight percent of patients had a good clinical outcome (modified Rankin scale between 0-2) at 3-6 month follow up.

Conclusion eCAS using the Casper stenting system is effective and technically feasible in patients presenting with acute stroke and concomitant carotid artery stenosis.

Disclosures A. Lamanna: None. J. Maingard: None. H. Kok: None. C. Barras: None. A. Jhamb: None. V. Thijs: None. R. Chandra: None. D. Brooks: None. H. Asadi: None.

\section{P-007 FIRST PASS EFFECT ANALYSIS USING THE TREVO STENT-RETRIEVER ACUTE STROKE (TRACK) REGISTRY}

${ }^{1} \mathrm{M}$ Mokin*, ${ }^{1} \mathrm{C}$ Primiani, ${ }^{2} \mathrm{~A}$ Castonguay, ${ }^{3} \mathrm{R}$ Nogueira, ${ }^{3} \mathrm{D}$ Haussen, ${ }^{4} \mathrm{~J}$ English, ${ }^{5} \mathrm{~S}$ Satti, ${ }^{6} \mathrm{~J}$ Chen, ${ }^{7} \mathrm{H}$ Farid, ${ }^{8} \mathrm{C}$ Borders, ${ }^{9} \mathrm{E}$ Veznedaroglu, ${ }^{9} \mathrm{M}$ Binning, ${ }^{10} \mathrm{~A}$ Puri, ${ }^{11} \mathrm{~N}$ Vora, ${ }^{11} \mathrm{R}$ Budzik, ${ }^{12} \mathrm{G}$ Dabus, ${ }^{12}$ I Linfante, ${ }^{13} \mathrm{~V}$ Janardhan, ${ }^{14} \mathrm{~A}$ Alshekhlee, ${ }^{15} \mathrm{M}$ Abraham, ${ }^{16} \mathrm{R}$ Edgell, ${ }^{17} \mathrm{M}$ Taqi, ${ }^{18} \mathrm{R}$ El Khoury, ${ }^{19} \mathrm{~A}$ Majjhoo, ${ }^{20} \mathrm{M}$ Kabbani, ${ }^{21} \mathrm{M}$ Froehler, ${ }^{22}$ I Finch, ${ }^{23}$ S Ansari, ${ }^{24} \mathrm{R}$ Novakovic, ${ }^{25} \mathrm{~T}$ Nguyen, ${ }^{26} \mathrm{O}$ Zaidat. ${ }^{1}$ University of South Florida, Tampa, FL; ${ }^{2}$ Biostatistics, UT Southwestern Medical Center, Dallas, TX; ${ }^{3}$ Neurology, Emory University School of Medicine, Atlanta, GA; ${ }^{4}$ Neurology, California Pacific Medical Center, San Francisco, CA; ${ }^{5}$ Neurointerventional Surgery, Christiana Care Health Center, Newark, DE; ${ }^{6}$ Sidney Kimmel Medical College, Philadelphia, PA; ${ }^{7}$ Neurointerventional Radiology, St Jude Medical Center, Fullerton, CA; ${ }^{8}$ University of California, Irvine School of Medicine, Irvine, $C A ;{ }^{9}$ Neurosurgery, Drexel Neurosciences Institute, Philadelphia, PA; ${ }^{10}$ Radiology, University of Massachusetts Medical School, Worcester, MA; ${ }^{11}$ Radiology, Riverside Radiology and Interventional Associates, Columbus, OH; ${ }^{12}$ Neurointerventional Surgery, Baptist Cardiac and Vascular Institute, Miami, FL; ${ }^{13}$ Texas Stroke Institute, Plano, TX; ${ }^{14}$ Vascular and Interventional Neurology, DePaul Stroke Center-SSM Neuroscience Institutes, St. Louis, MO; ${ }^{15}$ Neurology and Interventional Radiology, University of Kansas Medical Center, Kansas City, KS; ${ }^{16}$ Neurology, St Louis University, St. Louis, MO; ${ }^{17}$ Neurology and Neurosurger, Los Robles Hospital and Medical Center, Thousand Oaks, CA; ${ }^{18}$ Neurology, Tulane University, New Orleans, LA; ${ }^{19}$ Neurology, Wayne State School of Medicine, Detroit, Ml; ${ }^{20}$ Neurosurgery, Gundersen Health System, La Crosse, WI; ${ }^{21}$ Neurology, Neurosurgery, and Radiology, Vanderbilt University Medical Center, Nashville, $T N_{;}{ }^{22}$ John Muir Health, Walnut Creek, CA; ${ }^{23}$ Radiology, Neurology, and Neurological Surgery, Northwestern University, Feinberg School of Medicine, Chicago, IL; ${ }^{24}$ Radiology, Neurology, and Neurotherapeutics, UT Southwestern Medical Center, Dallas, TX; ${ }^{25}$ Neurology, Neurosurgery, and Radiology, Boston Medical Center, Boston, MA; ${ }^{26}$ Endovascular Neurosurgery and Stroke, St Vincent Mercy Medical Center, Toledo, $\mathrm{OH}$

\subsection{6/neurintsurg-2019-SNIS.43}

Background and objective The first pass effect (FPE) has become a novel measure for neurointerventionalists, achieving a complete recanalization with a single thrombectomy device pass, which has been shown to be associated with higher rates of good clinical outcomes. We describe and expand the FPE to one of a large US post-marketing registry (TRACK, Trevo Stent-Retriever Acute Stroke) to elucidate the effect of a single device pass and clinical outcome.

Methods TRACK was a core-laboratory adjudicated multicenter registry of 634 patients from 23 centers aimed to evaluate the use of the Trevo device in everyday clinical practice. The TRACK registry was utilized to identify an FPE subgroup $(n=140)$. Baseline features including demographics and clinical data were compared with the non-FPE patients $(n=469)$. Clinical outcome measures included modified Rankin Scale score (mRS) at 30 and 90 days, National Institutes of Health Stroke Scale (NIHSS) score, symptomatic intracranial hemorrhage $(\mathrm{sICH})$, and mortality. FPE was defined as a single pass/use of a device with TICI 2c/3 recanalization, and no use of rescue therapy.

Results 609 patients were included in the FPE (140/609, 23\%) vs non-FPE $(469 / 609,77 \%)$ subgroup analysis from the TRACK registry. There was no association between patient demographics and FPE group, including age $(p=0.36)$, sex $(p=0.50)$, race $(p=0.49)$, location of occlusion $(p=0.26)$, baseline NIHSS $(\mathrm{p}=0.61)$ past medical history including hypertension $(p=0.57)$, atrial fibrillation $(p=0.55)$, diabetes mellitus $(p=0.74)$, hyperlipidemia $(p=0.28)$, coronary artery disease $(p=0.27)$, or history of smoking $(p=0.12)$. The non-FPE group was associated with higher use of intraarterial tPA (FPE: $11 \%$ vs non-FPE: $23 \%$ ). The onset to puncture time in minutes was not significantly different between groups $(p=0.31)$; however, the total procedural time, total fluoroscopic time, and puncture to reperfusion time had significantly shorter mean time in FPE group ( $p<0.0001$ for all variables). Clinical outcomes based on mRS at 30 and 90 days were significantly in favor of the FPE group, $p=0.0069$ and $\mathrm{p}<0.0001$, respectively. Good clinical outcome (mRS 0-2 at 90 days) was achieved in $63 \%$ of FPE group (77/122) compared to non-FPE at $44 \%(177 / 398), p=0.0004$; furthermore, NIHSS at discharge was significantly lower in FPE group (9.1 $\pm 11.2)$ compared to non-FPE $(12 \pm 11), p=0.0004$. Distal emboli in the same territory (EDT) was lower in the FPE group $(1 / 104,1 \%)$ compared to non-FPE (110/371, 30\%), $\mathrm{p}<0.0001$; however, no association in embolization into new territory (ENT), sICH, or death $(0.99, \quad 0.85, \quad 0.79$, respectively).

Conclusion The achievement of complete revascularization from a single Trevo Stent-Retriever device pass is associated with higher rates of good clinical outcomes. Additionally, FPE in our subgroup registry analysis was associated with shorter procedural times, lower NIHSS at discharge, and significantly lower rates of EDT. Our analysis was not able to identify any patient demographics that would be associated to achieve first pass effect. Further research is warranted to confirm findings and identify additional factors achieving good clinical outcome after thrombectomy.

Disclosures M. Mokin: 2; C; Claret Medical, Nogueira-Stryker Neurovascular (Trevo-2 Trial Principal Investigator; DAWN Trial Principal Investigator, TREVO Registry Steering Committee), Medtronic (SWIFT Trial Steering Committee; SWIFTPrime Trial Steering Committee ; STAR Trial Angiographic Core Lab), Penumbra (3D Separator Trial Executive Committee), Neuravi (ARISE-2 Steering Committee), Genentech (Physician Advisory Board), Allm Inc (Physician Advisory Board). C. Primiani: None. A. Castonguay: None. R. Nogueira: None. D. Haussen: None. J. English: None. S. Satti: 2; C; Stryker Neurovascular. J. Chen: None. H. Farid: None. C. Borders: None. E. Veznedaroglu: None. M. Binning: None. A. Puri: None. N. Vora: None. R. Budzik: None. G. Dabus: None. I. Linfante: 2; C; Metronic, Stryker, Penumbra, and Cordis. V. Janardhan: None. A. Alshekhlee: None. M. Abraham: None. R. Edgell: None. M. Taqi: None. R. El Khoury: None. A. Majjhoo: None. M. Kabbani: None. M. Froehler: None. I. Finch: None. S. Ansari: None. R. Novakovic: None. T. Nguyen: None. O. Zaidat: 6; C; overall PI for TRACK, Arise II, Co-PI Therapy Trial, Steering committee STRATIS registry. 